\title{
Estudo do impacto de um curso MBA Gestão em Saúde na modernidade organizacional de clínicas of talmológicas
}

\author{
Study of the impact of a MBA Administration in Health course on the organizational \\ modernity of ophthalmologic clinics
}

Manuel Meireles ${ }^{1}$

Marinho Jorge Scarpi ${ }^{2}$

\section{RESUMO}

Objetivo: Pesquisa no campo da aprendizagem de competências que busca investigar o impacto da aquisição de novas competências individuais dos responsáveis de clínicas oftalmológicas, por meio de um curso MBA Gestão em Saúde, na modernidade organizacional de clínicas oftalmológicas. Métodos: Levantamento utilizando questionários aplicados a alunos e ex-alunos do curso e a gestores de clínicas oftalmológicas não vinculados ao curso ( $n=30$ cada um). A análise foi feita por meio de métodos estatísticos: r de Pearson e Q de Yule; testes t de Student, da soma de séries, da mediana e teste U de Mann-Whitney. Resultados: Observou-se correlação linear e positiva entre a excelência do MBA Gestão em Saúde e o desenvolvimento de capacidades dos alunos veteranos (correlação rho de Spearman em nível de 0,01); há diferença significativa $(0,0364$, pelo teste de Mann-Whitney), entre alunos veteranos e alunos calouros, quanto à necessidade de se ampliar as capacidades individuais que o curso MBA Gestão em Saúde fornece, aos demais funcionários operacionais das clínica oftalmológicas; e há diferença significativa (0,0057, pelo teste de Mann-Whitney), entre alunos veteranos do curso MBA Gestão em Saúde e alunos calouros quanto à importância da modernidade organizacional para as clínicas oftalmológicas. Conclusões: Os resultados obtidos são consistentes, de forma geral, com o modelo proposto: a excelência do MBA Gestão em Saúde contribui significativamente para a modernidade organizacional, para o desenvolvimento de competências dos alunos egressos e ainda de forma substancial para a percepção de hiatos organizacionais.

Descritores: Competência profissional; Aprendizado; Qualidade; Capacidade de gestão; Modernização organizacional; Setor privado/tendências

Trabalho realizado no Departamento de Oftalmologia da Universidade Federal de São Paulo (UNIFESP).

Doutor, Universidade Federal de São Paulo (UNIFESP). São Paulo (SP).

${ }^{2}$ Livre-docente, Universidade Federal de São Paulo (UNIFESP). São Paulo (SP).

Endereço para correspondência: Manuel Meireles Cx. Postal 110 - Araçariguama (SP) CEP 18147-970

E-mail: profmeireles@uol.com.br

Recebido para publicação em 28.07.2005

Versão revisada recebida em 15.08.2005

Aprovação em 10.09.2005

Não tem agência financiadora.

\section{INTRODUÇÃO}

A presente pesquisa tem como tema a aprendizagem de competências, especificamente a aprendizagem individual adquirida por meio do MBA Gestão em Saúde do Instituto da Visão e seu potencial impacto na modernidade organizacional. $O$ tema da aprendizagem individual vem adquirindo um especial interesse por parte dos pesquisadores ${ }^{(1)}$ que argumentam que as organizações que agora concorrem entre si por clientes e mercados, em escala jamais vista, têm sido levadas a competir também por um recurso elevado à categoria de o mais importante de todos: o talento humano.

O tema objeto da presente pesquisa, isto é, a aquisição de competências é também relevante. A relevância de um trabalho é expressa, em grande
\end{abstract}


parte, pelo próprio objetivo da pesquisa, porquanto ${ }^{(2)}$ o objetivo da pesquisa é "descobrir respostas para perguntas, por meio do emprego de processos científicos". Além da aplicabilidade e utilidade da pesquisa, os resultados da presente pesquisa são úteis por mais três aspectos significativos de cunho científico $^{(2)}$, uma vez que as relações entre a teoria e a pesquisa não se dão em apenas uma direção. A teoria estimula a pesquisa e aumenta o sentido de seus resultados; a pesquisa empírica, por outro lado, serve para verificar as teorias existentes e apresentar uma base para a criação de novas teorias.

O objetivo da presente pesquisa é (i) averiguar até que ponto a aquisição de novas competências individuais dos responsáveis de clínicas oftalmológicas (por meio do MBA Gestão em Saúde) eleva nestes a percepção de se estender tais competências aos demais funcionários operacionais da clínica. A pesquisa investiga também (ii) a relação entre os quatro construtos nos quais ela se fundamenta: Excelência do MBA Gestão em Saúde; Percepção de hiatos organizacionais; Desenvolvimento de competências e Modernidade organizacional.

\section{Aprendizagem individual}

A presente pesquisa está vinculada à relação entre aprendizagem individual e aprendizagem organizacional com reflexo no desenvolvimento de competências. A aprendizagem organizacional não é estática ${ }^{(3)}$ e pode ser entendida como vários processos através dos quais habilidades ou conhecimento são adquiridos por indivíduos e convertidos, através deles, para a organização. A aprendizagem é essencial para se atingir resultados desejados ${ }^{(4)}$ : algumas vezes, o conhecimento é novo e tem que ser criado do início, outras vezes o conhecimento já existe e tem que ser transferido para algum lugar na organização.

Observou-se que existe uma relação entre aprendizagem organizacional e "performance"(5-9): a orientação para aprendizagem é valiosa para a organização, uma vez que estimula o foco no entendimento e satisfação das necessidades dos clientes, não apenas as expressas mas também as latentes, por meio de novos produtos e serviços, bem como novas formas de atuar no negócio. Esta condição leva a organização a melhores resultados, tais como sucesso de novos produtos, retenção de clientes, crescimento superior e lucratividade. Somente a aprendizagem $^{(8)}$ capacita as empresas a manter uma vantagem competitiva a longo prazo por meio do processamento contínuo das informações de mercado em uma velocidade maior do que a dos concorrentes.

A aprendizagem é um processo de aquisição da capacidade de usar o conhecimento ${ }^{(10)}$, que ocorre como resultado da prática e da experiência crítica e que produz uma mudança relativamente permanente no comportamento. Esta definição em muito se assemelha à de Garvin ${ }^{(4)}$ que destaca que as organizações que aprendem devem estar capacitadas a criar, adquirir e transferir conhecimentos e em modificar seus comportamentos para refletir estes novos conhecimentos e "insight".

Apesar do incremento de estudos sobre o construto de aprendizagem no âmbito das organizações, apresentam-se, ainda, importantes lacunas de consenso em termos conceituais, operacionais e metodológicos sobre o tema ${ }^{(4,11-12)}$. Uma explicação plausível poderia estar associada ao fato de ser a aprendizagem um construto complexo e multidimensional, estabelecido em diferentes níveis cognitivos e, potencialmente, envolvendo múltiplos subprocessos ${ }^{(13)}$.

Cada construto utilizado na pesquisa implica no reconhecimento de um referencial teórico, numa perspectiva de compreender a realidade. São particularmente importantes, para a presente pesquisa os construtos seguintes.

\section{Qualidade do ensino}

Este construto busca avaliar a percepção do corpo discente sobre a qualidade de um serviço educacional por meio da aplicação do modelo SERVQUAL ${ }^{(14)}$ e adaptado ao serviço educacional $^{(15)}$. As dimensões da escala SERVQUAL para o serviço educacional, procuram atender às características do setor, permitindo a adequada mensuração e categorização de cada uma delas para o ambiente do ensino, a saber: a) confiabilidade: a capacidade de prestar o curso prometido de modo confiável e com precisão, satisfazendo as necessidades primárias dos alunos; b) tangibilidade: a aparência física das instalações, equipamentos, pessoas e demais materiais envolvidos na realização do curso; c) empatia: a atenção e o carinho individualizados proporcionados aos alunos; d) segurança: o conhecimento e a cortesia de empregados e sua habilidade em transmitir confiança e exatidão; e e) responsividade: a disposição para ajudar o aluno e proporcionar com presteza o serviço. Portanto, para o presente construto considerou-se o modelo proposto por Parasuraman et $\mathrm{al}^{(14)} \mathrm{com}$ as oportunas adaptações ao serviço educacional elaboradas por Boulding et $\mathrm{al}^{\left({ }^{(15)} \text {. }\right.}$

$\mathrm{O}$ conceito de Excelência do curso está vinculado à forma como um curso procede à desconfirmação das expectativas dos seus alunos. O serviço educacional ${ }^{(16)}$ é um serviço de ações intangíveis, dirigido à mente das pessoas, de entrega contínua, realizado por meio de uma parceria entre a organização de serviço e seu aluno, e que, apesar de proporcionar um alto contato pessoal, é de baixa customização. E este serviço não se resume apenas à atividade de aprendizagem mas também às demais dimensões dos serviços ${ }^{(17)}$.

Surpreendentemente até há pouco tempo, não havia uma preocupação evidente com a qualidade dos serviços de ensino $^{(18)}$. O interesse por conhecer melhor a qualidade e a organização interna de uma instituição de ensino (que pode ser determinante no desempenho dos profissionais dela oriundos) somente adquiriu fôlego no Brasil a partir dos anos 90, seguindo uma tendência que despontava nos EUA e na Europa ${ }^{(19)}$.

Pode ser facilmente constatado, no caso de serviços no âmbito do ensino, e particularmente do ensino superior, ou seja, o aluno "é uma entrada no processo do serviço", sendo portanto, em princípio, tão mais modificado quanto melhor for o serviço ${ }^{(18)}$. Essa preocupação em salientar tal distinção também se faz presente em outros autores ${ }^{(20-25)}$, e que, em geral, consideram que o setor de serviços quando comparado à indústria é distinto o suficiente para que tenha uma aborda- 
gem diferenciada. Além disto, consideram a necessidade de haver uma categorização dos serviços apontando a existência de serviços puros, não agregados a bens materiais (ex.: consultoria, serviço jurídico) e os serviços agregados, prestados com bens materiais (ex.: restaurantes, que oferecem alimentos e serviços). Os serviços puros podem até envolver produtos na sua prestação, mas eles são o principal, enquanto os produtos são meros agregados.

Um instrumento de avaliação adequado do ensino superior deve estar direcionado a: (1) identificação de deficiências da metodologia de ensino; (2) falhas de concepção ou estruturação do curso; (3) satisfação dos alunos e (4) adequação às suas necessidades reais ${ }^{(26)}$. Deve ser observado que todos estes aspectos referem-se a requisitos contemplados nas Normas ISO 9000, ou seja o suposto "ideário de Qualidade total" pode se adequar ao que seriam "aspectos centrais" do processo educacional.

\section{Desenvolvimento de competências}

Este é um construto que leva em conta os estudos sobre a identificação das competências individuais ${ }^{(27-32)}$ sintetizados por Sant'anna et $\mathrm{al}^{(33)}$. Estes autores adotaram a técnica de análise de conteúdo por categoria ${ }^{(34)}$ para formatar um elenco de quinze competências mais reiteradamente apontadas nos trabalhos revisados, a saber: (1) domínio de novos conhecimentos técnicos associados ao exercício do cargo ou função ocupada; (2) capacidade de aprender rapidamente novos conceitos e tecnologias; (3) criatividade; (4) visão de mundo, ampla e global; (5) capacidade de inovação; (6) capacidade de comunicação; (7) capacidade de relacionamento interpessoal; (8) capacidade de trabalhar em equipe; (9) autocontrole emocional; (10) capacidade de lidar com situações novas e inusitadas; (11) capacidade de lidar com incertezas e ambigüidades; (12) iniciativa de ação e decisão; (13) capacidade de comprometer-se com os objetivos da organização; (14) capacidade de gerar resultados efetivos; e (15) capacidade empreendedora.

O conceito de Desenvolvimento de competências privilegia a perspectiva de competências individuais. De acordo com o resultado de uma pesquisa ${ }^{(35)}$ o desenvolvimento de competências é um processo de ensino-aprendizagem que é capaz de eliciar: (1) a capacidade de atuar em equipe; (2) a capacidade de investigação; (3) a capacidade de elaboração de propostas e de novas idéias; (4) a capacidade de relacionar e associar; (5) a capacidade de atuar em interdisciplinaridade; (6) a capacidade de analisar e avaliar situações vividas por empresas; (7) a capacidade de aplicar metodologias - científicas e operacionais; (8) a capacidade de buscar informações e conteúdos do interesse da organização; (9) a capacidade de trocar experiências entre gestores de empresas e áreas diferentes; e (10) a capacidade de ampliar horizontes.

\section{Qualidade de serviços}

O construto Percepção de hiatos organizacionais estrutura-se no modelo conceitual de Qualidade de serviços ${ }^{(36)}$. O conceito de Percepções de hiatos organizacionais, está asso- ciado a esse modelo e à capacidade de perceber e avaliar causas potenciais de falhas de qualidade em serviços: (1) causas que permitem a existência de hiato entre as expectativas do cliente e a percepção do gestor quanto a essas expectativas; (2) causas que possibilitam a existência de hiatos entre as especificações do serviço e a percepção do gestor quanto às expectativas dos clientes; (3) causas que levam à existência de hiato entre o serviço entregue e as especificações da qualidade do serviço; (4) causas que possibilitam hiato entre o serviço entregue e a comunicação externa dirigida aos clientes ou potenciais clientes; e (5) causas que geram hiato entre as expectativas do cliente quanto ao serviço e a qualidade percebida pelo cliente quanto ao serviço recebido.

\section{Modernidade organizacional}

O construto Modernidade organizacional é aqui entendido de acordo com a abordagem de Análise dos padrões de Modernidade organizacional ${ }^{(37)}$, agregando perspectivas de vários autores ${ }^{(38-40)}$. Esta abordagem estabelece um conjunto significativo de indicadores de modernidade, abrangendo as dimensões cultural, política, social, administrativa, econômica e tecnológica das organizações.

Eboli $^{(37)}$ destaca que estabelecer os indicadores de modernidade organizacional constitui etapa fundamental para a realização de pesquisas nesta área, uma vez que somente a partir da identificação e seleção das principais variáveis pode-se avaliar de forma mais completa e abrangente a modernidade na gestão empresarial. A modernidade organizacional é impulsionada por fatores: (1) que valorizam a iniciativa, a responsabilidade e a liberdade; (2) que estimulam um clima interno que favorece mudanças, inovação e aprendizagem; (3) que conduzem a um regime democrático e a um processo decisório descentralizado; (4) que estimulam a autonomia, a iniciativa de ação e de decisão; (5) que encorajam a interação social e a participação das pessoas nos processos organizacionais; (6) que favorecem um nível de informação de tal forma que as pessoas atinjam seus objetivos, materiais ou psicológicos; (7) que conduzem à formulação clara da missão, dos objetivos, das estratégias e metas organizacionais; (8) que induzem a adoção de tecnologias, políticas e práticas que promovem a tomada de risco, a criatividade, a eficácia e o desempenho das pessoas; (9) que instiga a subordinação dos objetivos econômicos aos objetivos sociais e princípios éticos; e (10) que induz ao uso da tecnologia para favorecer a interação entre pessoas e áreas bem como a integração de tecnologias avançadas com a criatividade das pessoas.

\section{Objeto de estudo}

O objeto de estudo da presente pesquisa é a excelência do curso MBA Gestão em Saúde, já que se parte do pressuposto que tal excelência induz ao desenvolvimento de competências e eleva a percepção de hiatos organizacionais e conseqüentemente conduz à modernidade organizacional das clínicas oftalmológicas. 
O Instituto da Visão é um órgão suplementar da UNIFESPEPM que abrange o IPEPO (Instituto Paulista de Estudos e Pesquisa em Oftalmologia) e o Departamento de Oftalmologia da UNIFESP. O Instituto da Visão presta serviços oftalmológicos e reúne as vantagens de um serviço médico com alta tecnologia disponível em todas as unidades, com a capacitação médica do mais alto nível. Os serviços oftalmológicos podem ser prestados a todos que procurem o Instituto da Visão particularmente ou pelas Assistências Médicas: Blue Life, Cabesp, Medservice, Assistália e Gama. Através do Hospital São Paulo o Instituto da Visão atende também a vários convênios.

Além disso, o Instituto da Visão oferece também serviço de apoio psicológico, diagnóstico e terapêutico para pacientes com problemas visuais. $\mathrm{O}$ atendimento psicológico objetiva aliviar o sofrimento psicológico que freqüentemente acompanha o paciente portador de doença ocular crônica, visão residual ou cegueira uni ou bilateral; objetiva auxiliar os pacientes e seus familiares a enfrentar estas situações da melhor forma; são discutidos aspectos relacionados à doença e reações frente à mesma de acordo com as necessidades de cada paciente.

\section{MBA's para médicos nos Estados Unidos}

Parece adequado mostrar uma rápida panorâmica de cursos semelhantes existentes nos Estados Unidos que possuem grande preocupação com a formação de médicos administradores. A área de assistência médica, crescentemente turbulenta, está treinando doutores e estudantes de medicina objetivando redefinir suas habilidades. As mudanças observadas na área da saúde levou à necessidade de médicos se transformarem em médicos executivos ${ }^{(41)}$. Isso é obtido por meio de treinamento de médicos em gestão de negócios, de forma que eles possam servir como ligação entre o pessoal administrativo e o clínico. Como a atual integração de sistemas, combinaram-se funções clínicas e administrativas; os papéis gerenciais aumentaram para os médicos e isso levou ao aumento da demanda por cursos com o objetivo de treinar lideranças médicas. É esperado um crescimento no número de executivos médicos nos Estados Unidos, de forma a facilitar as relações do médico nos serviços de assistência médica ${ }^{(42)}$.

A transição de papéis clínicos para funções administrativas está desafiando os médicos administradiores ${ }^{(43)}$. Movendo-se para papéis predominantemente administrativos, os médicos enfrentam desafios bem diferentes daqueles para os quais foram treinados ${ }^{(44)}$. O médico administrador é considerado um recurso efetivo para uma organização na área da saúde, e seu novo papel requer competências distintas, tais como o modo de pensar gerencial, nova filosofia, e novas atitudes e comportamentos $^{(45)}$. Considerando que o treinamento clínico tradicional recebido pelos médicos contrasta com a gestão organizacional, poucos médicos estão preparados para as exigências administrativas ${ }^{(46)}$.

A área da assistência médica está se caracterizando pela crescente incerteza e turbulência ${ }^{(47)}$ : em um ambiente de mudança rápida, a flexibilidade é crítica para o sucesso de geren- tes e de organizações. Os médicos administradores também devem estar abertos a mudanças e devem saber lidar com as incertezas da gestão, tolerando a ambigüidade em situações administrativas. Estudantes de MBA exibem uma tolerância à ambigüidade mais elevada do que os médicos tradicionais.

As ciências administrativas estão representando um papel crescentemente proeminente nas organizações de serviços de saúde (OSS) ${ }^{(48)}$. Apesar de relatórios de mídia afirmarem que um número ascendente de médicos está adquirindo competências administrativas por meio de MBAs, nenhum recente estudo tinha verificado isso. $\mathrm{O}$ estudo de Larson et al ${ }^{(48)}$ mediu mudanças no número e natureza das afiliações aos programas de MBA's para pessoal da área médica nos Estados Unidos: o número destes programas passou de seis para 33 entre 1993 e 2001, e mais 17 escolas médicas estavam considerando a instalação de programas de MBA. Tais programas tentam complementar educação médica com educação de gestão de empresas. O crescimento nos números de programas de MBA e de estudantes indica crescente cooperação entre escolas médicas e empresariais e um crescente interesse em prover disciplinas de administração nos cursos de graduação de médicos.

\section{MÉTODOS}

A presente pesquisa pode ser caracterizada como um estudo de campo, realizado por meio da técnica de "survey". É também uma pesquisa de natureza descritiva, na medida em que atende à caracterização de levantamentos descritivos ${ }^{(4)}$ que é determinar a incidência e distribuição das características e opiniões de populações de pessoas, obtendo e estudando características presumivelmente representativas de tais populações.

\section{Sujeitos}

O estudo debruçou-se de forma preponderante sobre alunos e ex-alunos do MBA Gestão em Saúde, gestores de clínicas ou de consultórios oftalmológicos. Os sujeitos foram divididos em três grupos, cada um com n=30: Grupo 1 (veteranos): alunos que concluíram o MBA Gestão em Saúde ou estavam prestes a concluir; Grupo 2 (calouros): alunos acabados de ingressar no MBA Gestão em Saúde; e Grupo 3 (externos): médicos Administradores de clínicas oftalmológicas sem MBA em Administração

\section{Coleta de dados}

Foram utilizadas escalas tipo Likert com 11 pontos que permitem que o tratamento das variáveis seja feito considerando-as como contínuas ${ }^{(50)}$, embora isto não seja pacífico. Os dados provenientes de escala Likert são de natureza estritamente ordinal e lamenta que ainda hoje as variáveis de nível ordinal formem a base de muitos dos dados usados em modelos paramétricos, quando esse tipo de processo de agregação assume no mínimo um nível intervalar de mensuração ${ }^{(51)}$.

A coleta de dados foi feita por questionários especialmente projetados para cada tipo de amostra, isto é, cada grupo de 
respondentes recebeu um questionário composto de partes específicas.

O questionário 1 (Quadro 1) é o mesmo aplicado por Dutra et $\mathrm{al}^{(52)}$. (2002) e foi ligeiramente modificado para ser utilizado na presente pesquisa. Este questionário estrutura-se no modelo proposto por Parasuraman et $\mathrm{al}^{\left({ }^{(1)}\right)} \mathrm{com}$ as adaptações ao serviço educacional elaboradas por Boulding et $\mathrm{al}^{(15)}$. O questionário 1 está baseado no modelo SERVQUAL adaptado ao serviço educacional, sendo composto por 48 proposições sobre as quais os alunos opinarão dentro de uma escala tipo Likert com diferencial semântico de onze pontos. Foi testada a consistência interna deste questionário, utilizando o coeficiente Alfa de Cronbach ${ }^{(52)}$. O resultado obtido foi de 0,80 constituindo resultado satisfatório $^{(53)}$ (valores entre 0,60 e 0,80 ). Desta forma pode-se considerar esta escala como sendo consistente e validada.

O questionário 2 (Quadro 2), buscou aferir as competências individuais desenvolvidas pelo Curso. O questionário 2 para mensuração do nível de Competências individuais desenvolvidas, baseou-se em quinze proposições que foram selecionadas por meio da técnica de análise fatorial exploratória ${ }^{(33)}$.

O questionário 3 (Quadro 3), referente à capacidade dos respondentes reconhecerem e avaliarem Hiatos organizacionais, está baseada no modelo de Zeithaml et al ${ }^{(36)}$.

Para a identificação das competências individuais mais enfaticamente requeridas aos profissionais como resposta às de- mandas do atual ambiente dos negócios, esses autores adotaram como marco teórico uma extensa revisão das abordagens anglo-americana e francesa sobre a competência. Este questionário já foi analisado quanto às suas propriedades psicométricas de dimensionalidade e confiabilidade das escalas ${ }^{(33,54)}$.

O questionário 4 (Quadro 4) destinou-se a aferir a Modernidade organizacional pretendida pelos respondentes. Foi feita a análise da confiabilidade do questionário obtendo-se coeficientes Alfa de Cronbach ${ }^{(33)}$ iguais ou superiores a 0,70 estando, desta forma, em conformidade com o proposto por Hair Jr. et $\mathrm{al}^{(55)}$.

A análise dos questionários 2 e 4 contemplou também a análise da validade convergente - grau em que um dado fator se correlaciona positivamente com as outras medições da mesma escala -, e da validade discriminante (grau em que uma dada medida não se correlaciona com outras variáveis para os quais se supõe diferir) além da validade monológica (aderência entre os resultados obtidos e o previsto na literatura) ${ }^{(55-56)}$, tendo obtido tais análises resultados satisfatórios. Desta forma considera-se que os questionários utilizados pela presente pesquisa são validados.

\section{Procedimentos}

Os passos referentes à operacionalização da pesquisa, foram os seguintes: (1) obteve-se a lista de todos os alunos

Quadro 1. Questionário 1 utilizado para avaliar expectativas dos alunos quanto ao Curso

\begin{tabular}{|c|c|c|c|c|c|c|c|c|c|c|c|}
\hline \multirow{2}{*}{$\begin{array}{l}\text { Parte } 1 \\
\text { Por favor, faça uma avaliação do seu curso MBA Gestão em Saúde considerando as } \\
\text { proposições abaixo e a escala de notas à direita }\end{array}$} & \multicolumn{11}{|c|}{ Avaliação } \\
\hline & \multicolumn{6}{|c|}{ Discordo } & \multicolumn{5}{|c|}{ Concordo } \\
\hline \multicolumn{12}{|l|}{ Os professores estruturam suas aulas para encorajar interação entre os estudantes } \\
\hline \multicolumn{12}{|l|}{ Professores comunicaram suas idéias claramente e efetivamente } \\
\hline \multicolumn{12}{|l|}{ Professores mostraram respeito aos alunos } \\
\hline \multicolumn{12}{|l|}{ Professores equilibram teoria e prática em sala de aula } \\
\hline \multicolumn{12}{|l|}{ O prédio e piso são limpos } \\
\hline \multicolumn{12}{|l|}{ Professores vestem-se apropriadamente e têm boa aparência } \\
\hline \multicolumn{12}{|l|}{ A temperatura é uniformemente confortável por todo o prédio } \\
\hline \multicolumn{12}{|l|}{ Os materiais usados no ensino foram claros e sem erros } \\
\hline \multicolumn{12}{|l|}{ Professores deram aos estudantes atenção individual } \\
\hline \multicolumn{12}{|l|}{ Professores ajudaram estudantes com problemas pessoais e aconselhamento de carreira } \\
\hline \multicolumn{12}{|l|}{ Cursos foram bem ensinados } \\
\hline \multicolumn{12}{|l|}{ O "staff" (funcionários) garantiu o bom andamento dos cursos } \\
\hline \multicolumn{12}{|l|}{ Professores são preparados para dar aula } \\
\hline \multicolumn{12}{|l|}{ Professores tiveram experiência de ensino antes de ingressar no curso } \\
\hline \multicolumn{12}{|l|}{ Professores ajudaram alunos nos trabalhos do curso } \\
\hline \multicolumn{12}{|l|}{ Professores entregaram roteiros de aula (ou semelhante) a cada aula } \\
\hline \multicolumn{12}{|l|}{$\begin{array}{l}\text { Professores deram aconselhamento individual caso estudantes estivessem interessados em } \\
\text { explorar assuntos mais profundamente }\end{array}$} \\
\hline Professores responderam os questionamentos de forma completa e exata na mesma aula & & & & & & & & & & & \\
\hline Fonte: Dutra et al. (2002). Modificado & & & & & & & & & & & \\
\hline
\end{tabular}




\begin{tabular}{|c|c|c|c|c|c|c|c|c|c|c|c|}
\hline \multicolumn{12}{|c|}{ Quadro 4. Questionário referente à modernidade organizacional } \\
\hline \multirow{3}{*}{$\begin{array}{l}\text { Parte } 3 \\
\text { Considerando que você é responsável por uma Clínica oftalmológica, por favor, informe } \\
\text { qual é a importância que atribui a cada proposição abaixo para a sua Clínica. Utilize a } \\
\text { escala de notas à direita. }\end{array}$} & \multicolumn{11}{|c|}{ Avaliação } \\
\hline & \multicolumn{6}{|c|}{ Baixa } & \multicolumn{5}{|c|}{ Alta } \\
\hline & 0 & 1 & 2 & 3 & 4 & 5 & 6 & 7 & 8 & 9 & 10 \\
\hline \multicolumn{12}{|l|}{1 - O sistema de remuneração da organização recompensa os atos de competência } \\
\hline \multicolumn{12}{|l|}{2 - A organização é fortemente orientada para resultados } \\
\hline \multicolumn{12}{|l|}{3 - Há um sistema de avaliação que permite diferenciar o bom e o mau desempenho } \\
\hline \multicolumn{12}{|l|}{$\begin{array}{l}4 \text { - A organização equilibra adequadamente a preocupação com resultados financeiros, com } \\
\text { as pessoas e com a inovação }\end{array}$} \\
\hline \multicolumn{12}{|l|}{$\begin{array}{l}5 \text { - As políticas e práticas de recursos humanos estimulam as pessoas a se preocuparem } \\
\text { com a aprendizagem contínua }\end{array}$} \\
\hline \multicolumn{12}{|l|}{6 - Os principais critérios para promoção são a competência e a produtividade da pessoa } \\
\hline \multicolumn{12}{|l|}{$\begin{array}{l}7 \text { - A organização combina de forma equilibrada a utilização de tecnologias avançadas com a } \\
\text { criatividade das pessoas }\end{array}$} \\
\hline \multicolumn{12}{|l|}{8 - A tecnologia empregada favorece a interação entre pessoas e áreas } \\
\hline \multicolumn{12}{|l|}{$\begin{array}{l}9 \text { - As políticas e práticas da organização estimulam que as pessoas estejam sempre bem } \\
\text { informadas e atualizadas }\end{array}$} \\
\hline \multicolumn{12}{|l|}{10 - A estratégia, missão, objetivos e metas da organização são claramente definidos } \\
\hline \multicolumn{12}{|l|}{$\begin{array}{l}11 \text { - As políticas e práticas de recursos humanos da organização estimulam o desenvolvimento } \\
\text { pessoal e profissional }\end{array}$} \\
\hline \multicolumn{12}{|l|}{$\begin{array}{l}12 \text { - De modo geral, os empregados sabem o que devem fazer para colaborar com os objetivos } \\
\text { da organização }\end{array}$} \\
\hline \multicolumn{12}{|l|}{13 - O processo decisório na organização é descentralizado } \\
\hline \multicolumn{12}{|l|}{14 - A organização favorece a autonomia para tomar decisões } \\
\hline \multicolumn{12}{|l|}{$\begin{array}{l}15 \text { - No que se refere ao aspecto político, o regime que vigora na organização pode ser } \\
\text { caracterizado como democrático }\end{array}$} \\
\hline \multicolumn{12}{|l|}{16 - Os processos de tomada de decisão são participativos e transparentes } \\
\hline \multicolumn{12}{|l|}{$\begin{array}{l}17 \text { - A organização conta com sistemas de gestão participativos que estimulam a iniciativa e } \\
\text { ação das pessoas }\end{array}$} \\
\hline \multicolumn{12}{|l|}{18 - A organização admite a diversidade de comportamentos e respeita as diferenças individuais } \\
\hline \multicolumn{12}{|l|}{$\begin{array}{l}19 \text { - O ambiente de trabalho facilita o relacionamento entre as pessoas, mesmo de níveis } \\
\text { hierárquicos diferentes }\end{array}$} \\
\hline \multicolumn{12}{|l|}{20 - O clima interno da organização estimula idéias novas e criativas } \\
\hline \multicolumn{12}{|l|}{$\begin{array}{l}21 \text { - O clima interno da organização estimula que as pessoas estejam em contínuo processo } \\
\text { de aprendizagem, no seu dia-a-dia de trabalho }\end{array}$} \\
\hline \multicolumn{12}{|l|}{$\begin{array}{l}22 \text { - Na organização há um clima estimulante para que as pessoas realizem suas atividades, } \\
\text { buscando se superar }\end{array}$} \\
\hline \multicolumn{12}{|l|}{23 - A organização encoraja a iniciativa e responsabilidade individual } \\
\hline \multicolumn{12}{|c|}{$\begin{array}{l}\text { Considerando as } 23 \text { proposições acima numeradas, informe qual é a que, a seu ver, é a mais importante de todas. } \\
\text { Escreva o número da proposição à direita. }\end{array}$} \\
\hline Fonte: Sant'anna et al. (2002), adaptado & & & & & & & & & & & \\
\hline
\end{tabular}

a averiguar se existe correlação linear e positiva entre duas variáveis; b) testes t de Student, da soma de séries, da mediana e teste U de Mann-Whitney, para verificar se há diferença significativa entre duas variáveis. A análise fez uso dos softwares SPSS v12; Minitab v13, KyPlot 2.0 e Grapf Pad InStat 3. Sempre que aplicável fez-se uso de estatísticas descritivas, tais como média, mediana e desvio padrão.

Análises do nível de aprovação: $\mathrm{O}$ aspecto mais problemático do uso das escalas não se prende à forma de apresentação, mas sim, à sua análise. "Interpretar" corretamente o que as escalas indicam tem uma grande importância para a presente pesquisa, pois possibilita entender as opiniões dos respondentes. Metade do valor central ou indiferente é atribuído para aprovação e metade para desaprovação ${ }^{(57)}$. Desta forma e considerando que os questionários utilizam notas de 0 a 10 , o porcentual de desaprovação considera a soma dos porcentuais das notas de 0 a 4 mais $50 \%$ da nota 5; o porcentual de aprovação considera a soma dos porcentuais das notas de 6 a 10 mais $50 \%$ da nota 5 . Assim esta análise permite que se sintetize a avaliação dos respondentes, mas desloca a resposta para um conceito bipolar: aprovação versus desaprovação sem uma interpretação intermediária ou mais objetiva.

Com vistas a averiguar se existe a correlação linear e positiva entre duas variáveis utilizaram-se os testes $r$ de Pearson, $Q$ de Yule ${ }^{(58)}$ e Coeficiente de correlação por postos de Sperman. Para verificar se há diferença significativa entre duas variáveis foram utilizados os testes t de Student (quando aplicáveis ou com função orientativa), da soma de séries, da mediana e teste U de Mann-Whitney. 


\section{RESULTADOS}

Os testes das hipóteses foram realizados utilizando-se testes não-paramétricos e, sempre que possível, desde que as amostras tivessem passado no teste de normalidade, testes paramétricos.

\section{Hipóteses não rejeitadas ao nível de significância de 0,05}

As seguintes hipóteses foram testadas e não rejeitadas:

1) Existe correlação linear e positiva entre a Excelência do MBA Gestão em Saúde e o Desenvolvimento de capacidades dos alunos veteranos. As correlações observadas foram: $r$ de Pearson ao nível de 0,01 e correlação rho de Spearman ao nível de 0,01 . Esperava-se que realmente se observasse uma correlação linear e positiva entre a Excelência do MBA Gestão em Saúde e o Desenvolvimento de capacidades dos veteranos. Tal hipótese foi confirmada.

2) Há diferença significativa entre alunos veteranos e alunos calouros, do MBA Gestão em Saúde quanto à necessidade de se ampliar as capacidades individuais que o curso MBA Gestão em Saúde fornece, aos demais funcionários operacionais da Clínica oftalmológica. Observou-se que existe diferença significativa ao nível de significância 0,0364 , pelo teste Mann-Whitney. Esta hipótese parece confirmar que a aprendizagem de competências individuais dos responsáveis pelas clínicas oftalmológicas eleva nestes a percepção de se estender tais competências aos demais funcionários operacionais da clínica.

3) Há diferença significativa entre alunos veteranos do MBA Gestão em Saúde e Médicos administradores (externos), quanto à necessidade de se ampliar as capacidades individuais que um curso de desenvolvimento de capacidades (MBA ou semelhante) fornece, aos demais funcionários operacionais da Clínica oftalmológica. Observou-se que existe diferença significativa ao nível de significância 0,0057 , pelo teste Mann-Whitney. Esta hipótese complementa o resultado do teste anterior.

4) Há diferença significativa entre alunos veteranos do Curso MBA e alunos calouros quanto à importância da modernidade organizacional para as clínicas oftalmológicas. A análise mostrou que existe diferença significativa ao nível de significância 0,0001 , pelo teste Mann-Whitney. A não rejeição deste hipótese parece reafirmar a importância do MBA Gestão em Saúde para a modernidade organizacional.

\section{Hipótese com evidência sugestiva}

Quando o "p-value" apresenta um valor entre 0,05 e 0,10 pode-se afirmar que há uma evidência sugestiva contra a hipótese nula ${ }^{(59)}$. Desta forma pode-se considerar a hipótese abaixo como ainda expressiva.

5) A hipótese de que há diferença significativa entre alunos calouros e alunos veteranos do MBA Gestão em Saúde quanto à Percepção de hiatos organizacionais é rejeitada ao nível de significância de 0,05 mas apresenta evidência suges- tiva contra a hipótese nula ao nível de significância 0,0514 pelo teste Mann-Whitney. Esta hipótese testa a relação entre a excelência do MBA Gestão em Saúde e a percepção de hiatos organizacionais sob o pressuposto lógico de que os veteranos teriam uma percepção maior dos hiatos do que os calouros. O valor do p-value observado sugere que o MBA Gestão em Saúde deva enfatizar esta relação.

\section{Hipóteses rejeitadas}

As hipóteses abaixo foram testadas e rejeitadas:

6) Rejeitada a hipótese de que existe correlação linear e positiva entre o desenvolvimento de capacidades dos alunos veteranos do MBA Gestão em Saúde e a modernidade organizacional pretendida para as clínicas oftalmológicas. É rejeitada ao nível de 0,335 de acordo com rho de Spearman. Esperava-se que o desenvolvimento de capacidades dos veteranos se expressasse por uma busca maior de modernidade organizacional e isso não se verificou

7) Rejeitada a hipótese de que há diferença significativa entre veteranos do MBA Gestão em Saúde e Médicos Administradores (externos) de clínicas oftalmológicas sem graduação em Administração ou MBA, quanto à Percepção de hiatos organizacionais, pois não existe diferença significativa ao nível de significância 0,8245, pelo teste Mann-Whitney. Este resultado foi surpreendente e não se dispõe de pesquisas adicionais capazes de explicar por que motivo tal hipótese não se verifica. Uma possível explicação pode residir numa possível maior experiência dos externos: os externos são responsáveis por clínicas oftalmológicas com um maior número de anos de existência.

8) Foi rejeitada a hipótese de que não há diferença significativa entre alunos calouros do MBA Gestão em Saúde e Médicos Administradores (externos) de clínicas oftalmológicas sem graduação em Administração ou MBA, quanto à Percepção de hiatos organizacionais. Esta hipótese é rejeitada ao nível de significância de 0,05 . A diferença é significativa ao nível de significância 0,0062, pelo teste Mann-Whitney. A lógica da pesquisa considerou calouros e externos no mesmo nível de percepção de hiatos organizacionais.

\section{COMENTÁRIOS}

A figura 1 mostra o modelo construído após a análise dos dados. Tal modelo centra-se em quatro construtos: Excelência do MBA Gestão em Saúde; Percepção de hiatos organizacionais; Desenvolvimento de competências e Modernidade organizacional, porém a vinculação entre a Percepção dos hiatos organizacionais e a Modernidade organizacional não se verificou. Pelo contrário: observou-se uma associação muito significativa (ao nível de significância <0,0001) entre Percepção de hiatos organizacionais e Desenvolvimento de competências.

Este resultado sugere que o curso de MBA Gestão em Saúde deva chamar a atenção para modelo conceitual de Qualidade de serviços ${ }^{(36)}$, especialmente quanto à capacidade de 


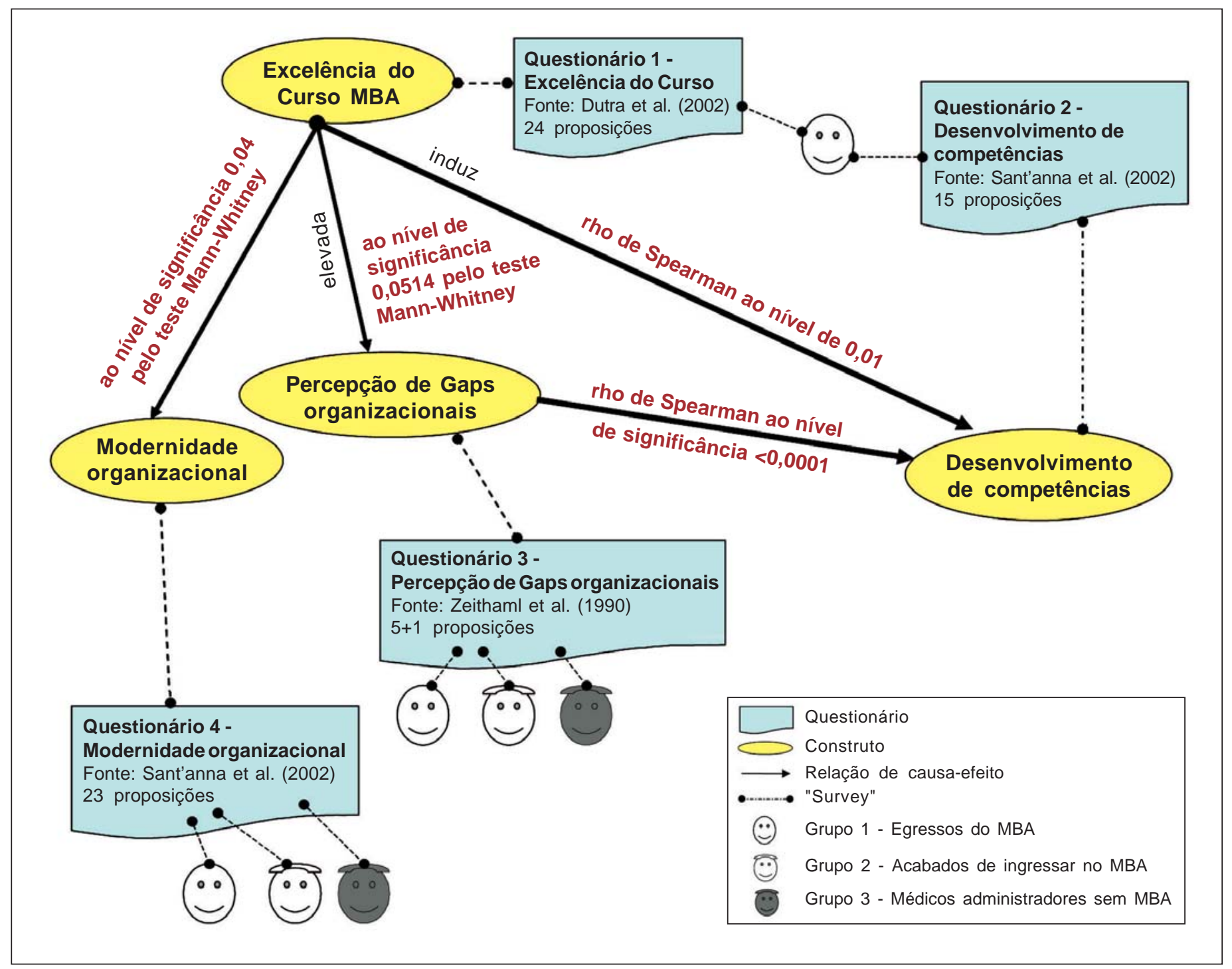

Figura 1 - A excelência do curso tem relação causal direta com a modernidade organizacional

perceber e avaliar causas potenciais de falhas de qualidade em serviços. O resultado observado também corrobora pesqui$\mathrm{sa}^{(60)}$ de Yamamoto (2004), que encontrou hiatos num conjunto de clínicas oftalmológicas numa proporção elevada: $82,0 \%$.

Os resultados tendem a evidenciar que a aprendizagem de competências individuais dos responsáveis pelas clínicas oftalmológicas eleva nestes a percepção de se estender tais competências aos demais funcionários operacionais da clínica. Ou seja: a aprendizagem individual de competências por um sujeito, dentro da organização, leva-o a buscar ampliar as competências para os demais membros da organização.

Em que pesem as limitações expostas, a conclusão extraída da pesquisa é que o MBA Gestão em Saúde contribui significativamente para o processo de aprendizagem - um processo de aquisição da capacidade de usar o conhecimento, que ocorre como resultado da prática e da experiência crítica e que produz uma mudança relativamente permanente no comportamento ${ }^{(10)}$.
Dado que as organizações que aprendem passam a estar capacitadas a criar, adquirir e transferir conhecimentos e em modificar seus comportamentos para refletir estes novos conhecimentos e "insight"(4), a modernidade organizacional passa a ser buscada, significando que os alunos egressos do MBA Gestão em Saúde: (a) valorizam a iniciativa, a responsabilidade e a liberdade; (b) estimulam um clima interno que favorece mudanças, inovação e aprendizagem; (c) conduzem a cultura organizacional a um regime democrático e a um processo decisório descentralizado; (d) estimulam a autonomia, a iniciativa de ação e de decisão; (e) encorajam a interação social e a participação das pessoas nos processos organizacionais; (f) favorecem um nível de informação de tal forma que as pessoas atinjam seus objetivos, materiais ou psicológicos; (g) conduzem à formulação clara da missão, dos objetivos, das estratégias e metas organizacionais; (h) induzem a adoção de tecnologias, políticas e práticas que promovem a tomada de risco, a criatividade, a 
eficácia e o desempenho das pessoas; (i) instigam a subordinação dos objetivos econômicos aos objetivos sociais e princípios éticos; (j) e induzem ao uso da tecnologia para favorecer a interação entre pessoas e áreas, bem como a integração de tecnologias avançadas com a criatividade das pessoas.

\section{CONCLUSÃO}

1 - Os resultados sugerem que a aquisição de novas competências individuais dos responsáveis de Clínicas oftalmológicas (por meio do MBA), eleva nestes a percepção de se estender tais competências aos demais funcionários operacionais da clínica;

2 - Confirmou-se também a correlação linear e positiva entre a Excelência do MBA e o Desenvolvimento de capacidades dos alunos egressos; e a existência de diferença significativa entre alunos egressos do Curso MBA e alunos acabados de ingressar quanto à importância da modernidade organizacional para as clínicas oftalmológicas;

3 - Os resultados obtidos são consistentes, de forma geral, com o modelo proposto: a Excelência do MBA Gestão em Saúde, contribui significativamente para a Modernidade organizacional, para o Desenvolvimento de competências dos alunos egressos, e, ainda de forma substancial para a Percepção de hiatos organizacionais.

\section{ABSTRACT}

Purpose: Investigations in the field of the learning competence that investigate the impact of the acquisition of new individual competence of those responsible for ophthalmologic clinics, by means of a MBA Administration in Health course, on the organizational modernity of ophthalmologic clinics. Methods: Survey using questionnaires applied to students and former students of the course and to managers of ophthalmologic clinics not linked to the course ( $\mathrm{n}=30$ each one). The analysis was made by means of statistical methods: $r$ of Pearson and $Q$ of Yule; Student's t test, sum of series test, median test and U test of Mann-Whitney. Results: Linear and positive correlation was observed between excellency of MBA Administration in Health and the development of the veteran students' capacities (rho correlation of Spearman at the level of 0.01); there is a significant difference ( 0.0364 , by the Mann-Whitney test), between veteran students and freshmen regarding the need to extend the individual capacities provided by the MBA Administration in Health course, to the other operational employees of the ophthalmologic clinics; and there is a significant difference $(0.0057$, for the Mann-Whitney test), among veteran students of the MBA Administration in Health course and freshmen regarding the importance of the organizational modernity for the ophthalmologic clinics. Conclusions: The obtained results are consistent, in a general way, with the proposed model: MBA Administration's excellence in Health contributes significantly to the organizational modernity, to the development of competence of the veteran students, and, also in substantial way to the perception of organizational hiatuses.

Keywords: Professional competence; Learning; Quality; Management capacity; Organizational modernization; Private sector/trends

\section{REFERÊNCIAS}

1. Bartlett CA, Ghoshal S. Uso das subsidiárias para uma estratégia de alcance global. In: Montgomery CA, Porter ME. Estratégia: a busca da vantagem competitiva. Rio de Janeiro: Campus; 1998.

2. Selltiz C. Métodos de pesquisa nas relações sociais. São Paulo: Epu: EDUSP; 1975

3. Bell M. Learning and the accumulation of industrial technological capacity in developing countries. In: Fransman M, King K. Technological capability in the Third World. New York: St Martin's; 1984.

4. Garvin DA. Managing quality: the strategic and competitive edge. New York: The Free Press; 1993.

5. Day GS. The capabilities of market-driven organizations. J Mark. 1994;58(4): 37-52.

6. Sinkula JM. Market information processing and organizational learning. J Mark. 1994;58(1):35-45.

7. Slater SF, Narver JC. Market Oriented Is Not Enough. In: Deshpandé R (ed). Developing a market orientation. Thousand Oaks: Sage; 1999.

8. Dickson PR. The static and dynamic mechanics of competition: a comment on Hunt and Morgan's comparative advantage theory. J Mark. 1996;60(3):102-6.

9. Sampaio CH, Perin MG. O Processo de Aprendizagem Organizacional e a Performance Empresarial: o Caso da Indústria Eletroeletrônica do Brasil. In: Anais do XXVII Congresso Enanpad. Rio de Janeiro: Anpad; 2003.

10. Caravantes GR, Bretas Pereira M. Aprendizagem organizacional versus estratégia de mudança organizacional planejada: um confronto crítico. Porto Alegre: FAPERGS; 1985.

11. Lipshitz R, Popper M, Oz S. Building learning organizations: The design and implementation of organizational learning mechanisms. Jf Appl Behavioral Science. 1996;32(3):292-303.

12. Popper M, Lipshitz R. Organizational learning: mechanisms, culture, and feasibility. ManageLearn. 2000;31(2):181-96.

13. Slater SF, Narver JC. Market orientation and the learning organization. J Mark. 1995;59:63-74.

14. Parasuraman A, Berry LL, Zeithaml VA. Delivering quality service. New York; London : Free Press: Collier Macmillan; 1991.

15. Boulding W, Kaira A, Staehn A, Zeithaml V. A dynamic process model of service quality: From expectations to behavioral intentions. J Mark Res. 1993; 30:7-27.

16. Lovelock $\mathrm{CH}$. Classifying services to gain strategic marketing insights. J Mark. 1983;47:9-20.

17. Morales M, Calderón LF. Assessing service quality in schools of business: dimensions of service in continuing professional education (CPE). BALAS Latin America's New Millennium Proceedings; 1999. p.524-36.

18. Quintella RH, Melo PV, Leal R. Qualidade em Serviços Educacionais: o Caso da Certificação ISO 9002 do Curso de Especialização em Administração da UFBA. In: Anais. XXV Congresso Enanpad. Rio de Janeiro: Anpad; 2001

19. Xavier ACR. A Gestão da qualidade e a excelência dos serviços educacionais: custos e benefícios de sua implantação [texto na Internet]. In: Instituto de Pesquisa Econômica Aplicada - IPEA [sítio na Internet]. Brasília (DF): IPEA; 1996 [citado 2005 jan 7]. Disponível em http://www.ipea.gov.br

20. Albrecht K. Serviços com qualidade: a vantagem competitiva. São Paulo: Makron Books; 1992.

21. Albrecht K. Única coisa que importa: trazendo o poder do cliente para o centro de sua empresa. Traduzido por Montingelli Jr. N. São Paulo: Pioneira; 1993.

22. Normann R. Administração de serviços: estratégia e liderança na empresa de serviços. São Paulo: Atlas; 1993.

23. Lamprecht JL. ISO 9000 e setor de serviços: uma interpretação crítica das revisões de 1994. Rio de Janeiro: Qualitymark; 1994.

24. Berry LL. Serviços de satisfação máxima: guia prático de ação. Rio de Janeiro: Campus; 1996.

25. Desatnick RL, Detzel DH. Gerenciar bem é manter o cliente. São Paulo: Pioneira; 1994. 
26. Bandeira ML, Gonçalves CA, Veiga RT, Huertas MKZ. Avaliação da qualidade do ensino de pós-graduação: elementos para a construção e validação de um instrumento de pesquisa. In: Anais. XXII Congresso Enanpad. Rio de Janeiro: Anpad; 1998.

27. McClelland DC, Dailey C. Improving officer selection for the foreign service. Boston: McBer; 1972.

28. Spencer LM, Spencer S. Competence at work. New York: John Wiley; 1993.

29. Le Bortef G. De la compétence: essai sur un attracteur étrange. Paris: Les Editions de L'Organizations; 1995.

30. Stroobants M. Savoir-faire et compétence au travail: une sociologie de la fabrication des aptitudes. Bruxelles: Éditions de l'Université de Bruxelles; 1993

31. Zarifian P. Objetivo competência por uma nova lógica. São Paulo: Atlas; 2001.

32. Perrenoud P. Ensinar: agir na urgência, decidir na incerteza. Porto Alegre: Artmed; 2001.

33. Sant'anna AS, Moraes LFR, Kilimnik ZM. Competências individuais requeridas, modernidade organizacional e satisfação no trabalho: uma análise de organizações mineiras sob a ótica de profissionais da área de administração. In: Anais. XXVI Congresso Enanpad. Rio de Janeiro: Anpad, 2002. 34.

34. Richardson RJ. Pesquisa social: métodos e técnicas. São Paulo: Atlas; 1985

35. Ruas R. Desenvolvimento de competências gerenciais e contribuição da aprendizagem organizacional. In: Fleury MTL, Oliveira Jr MM. Gestão estratégica do conhecimento: integrando aprendizagem, conhecimento e competências. São Paulo: Atlas; 2001.

36. Zeithaml A, Parasuraman A, Berry L. Delivering Quality Service - Balance customer perceptions and expectations. New York: The Free Press; 1990.

37. Eboli MP. Modernidade na gestão de bancos (Tese de Doutorado). Universidade de São Paulo. Faculdade de Economia Administração e Contabilidade. São Paulo; 1996.

38. Motta R. A busca da competitividade nas empresas. RAE. 1995;35(2);12-6.

39. Faoro R. A questão nacional: a modernização. Estud Av. 1992;6(14):7-22.

40. Zajdsznajder L. Pós-modernidade e tendências da administração contemporânea. Bol Téc Senac. 1993;19(3);10-9.

41. Sherrill WW. Dual-degree MD-MBA students: a look at the future of medical leadership. Acad Med. 2000;75(10 Suppl):S37-9.

42. Smallwood KG, Wilson CN. Physician - executives past, present, and future. South Med J. 1992;85(8):840-4. Review.

43. Peters RM. When physicians fail as managers: an exploratory analysis of career change problems. Tampa: American College of Physician Executives, 1994.
44. Hagland MM. Physician executives bring clinical insight to non-clinical challenges. Hospitals. 1991;65(18):42,44,46-8

45. Kurtz M. The Dual Role Dilemma in new leadership in health care management. Tampa, FL: American College of Physician Executives; 1992.

46. Ott JE. Administrative medicine. JAMA. 1992;268(3);332-3.

47. Sherrill WW. Tolerance of ambiguity among MD/MBA students: implications for management potential. J Contin Educ Health Prof. 2001;21(2):117-22.

48. Larson DB, Chandler M, Forman HP. MD/MBA programs in the United States: evidence of a change in health care leadership. Acad Med. 2003;78(3): 335-41.

49. Kelinger FN. Metodologia da pesquisa em ciências sociais: um tratamento conceitual. São Paulo: Epu; 1980.

50. Gonçalves Filho C, Guerra RS, Moura A. Mensuração de Satisfação, Qualidade, Lealdade, Valor e Expectativa em Instituições de Ensino Superior: um estudo do modelo ACSI através de Equações Estruturais. In: Anais. XXVII Congresso Enanpad. Rio de Janeiro: Anpad; 2003.

51. Clogg CC, Shihadeh. Statistical models for ordinal variables: advanced quantitative techniques in the social sciences 4. Califórnia: Sage Publications; 1994.

52. Dutra HFO, Oliveira PAS, Gouveia TB. Avaliando a Qualidade de Serviço numa Instituição de Ensino Superior. In: Anais. XXVI Congresso Enanpad. Rio de Janeiro: Anpad, 2002.

53. Churchill Jr G. Marketing research - methodological foundations. Orlando: The Dryden Press; 1995.

54. Oliveira-Castro GA. Percepção de suporte organizacional: desenvolvimento e validação de um questionário. In: Anais. XXII Congresso Enanpad. Rio de Janeiro: Anpad; 1998

55. Hair Jr JF, et al. Multivariate data analysis. 5th ed. Upper Saddle River, N.J.: Prentice Hall; 1998.

56. Bagozzi RP, Yi Y, Phillips LW. Assessing construct validity in organizational research. Administrative Science Quaterly, Cornell. 1991;36:421-58.

57. Pereira JCR. Análise de dados qualitativos: estratégias metodológicas para as ciências da saúde, humanas e sociais. São Paulo: EDUSP; 1999.

58. Davis JA. Levantamento de dados em sociologia: uma análise estatística elementar. Rio de Janeiro: Zahar; 1976.

59. Arsham H. Razonamiento estadístico para la tomada de decisiones gerenciales. Madrid: PHA; 2001

60. Yamamoto EY. Disfunção competitiva: uma contribuição à gestão da estratégia de serviços das clínicas oftalmológicas [tese]. Universidade Federal de São Paulo. Escola Paulista de Medicina. Ciências da Saúde. São Paulo; 2004. 